\title{
B-Pol: detecting primordial gravitational waves generated during inflation
}

\author{
Paolo de Bernardis • Martin Bucher • \\ Carlo Burigana • Lucio Piccirillo • \\ For the B-Pol Collaboration
}

Received: 7 January 2008 / Accepted: 4 August 2008 / Published online: 28 August 2008

(c) Springer Science + Business Media B.V. 2008

\begin{abstract}
B-Pol is a medium-class space mission aimed at detecting the primordial gravitational waves generated during inflation through high accuracy measurements of the Cosmic Microwave Background polarization. We discuss the scientific background, feasibility of the experiment, and implementation developed in response to the ESA Cosmic Vision 2015-2025 Call for Proposals.
\end{abstract}

Keywords Cosmology $\cdot$ Cosmic microwave background $\cdot$ Satellite

See http://www.b-pol.org for the full list of collaboration members and a full copy of the B-Pol proposal.

\footnotetext{
P. de Bernardis $(\bowtie)$

Dipartimento di Fisica, Università di Roma La Sapienza,

P.le A. Moro 2, 00185, Roma, Italy

e-mail: paolo.debernardis@roma1.infn.it

M. Bucher

Laboratoire de Physique Théorique, Université Paris XI,

Bâtiment 210, 91405 ORSAY Cedex, France

e-mail: martin.bucher@th.u-psud.fr

C. Burigana

IASF-Bologna, INAF, Via Gobetti 101, 40129, Bologna, Italy

e-mail: carlo.burigana@iasfbo.inaf.it

\section{Piccirillo}

School of Physics and Astronomy, University of Manchester,

Oxford Road, Manchester, M13 9PL, United Kingdom

e-mail: lucio@jb.man.ac.uk
} 


\section{B-Pol science}

The quest to understand the origin of the tiny fluctuations about a perfectly homogeneous and isotropic universe lies at the heart of both modern cosmology and high-energy physics. Inflationary theory offers the most satisfying and plausible explanation for the initial conditions of the universe. Inflation is a phase of superluminal expansion of space itself, within $10^{-35} s$ of the Big Bang, during which quantum fluctuations are stretched to cosmological scales (see e.g., [1-10]). Results from Cosmic Microwave Background (CMB) experiments have established that the universe is almost spatially flat, with a nearly Gaussian, scale-invariant spectrum of primordial adiabatic perturbations (see e.g., [11-14]). These features are all consistent with the simplest models of inflation. By providing accurate measurements of the E-mode (gradient component) polarization of the CMB, the ESA mission Planck will offer more stringent tests of the inflationary paradigm [15]. Nevertheless, even with such an accurate characterization of the scalar perturbations, a decisive confirmation of inflation will be lacking and large uncertainties in the allowed inflationary potentials will persist. Inflation predicts the existence of primordial gravitational waves on cosmological scales. Their detection would firmly establish the existence of a period of inflationary expansion in the early universe, and confirm the quantum origin of cosmological fluctuations that led to the large scale structure observed today. The search for primordial B-mode (curl component) polarization of the $\mathrm{CMB}$ provides the only opportunity to detect in the foreseeable future the imprint of these gravitational waves. Measuring the amplitude of these tensor perturbations at one length scale would fix the energy scale of inflation and its potential. Measuring their amplitude at more than one length scale would provide a powerful consistency check for a broad class of inflationary models. If as suggested by recent CMB and large scale galaxy surveys, the power spectrum of primordial perturbations is not exactly scale invariant, then in a wide class of inflationary models the level of gravitational waves will be within the range accessible to a properly designed mission, as shown in Fig. 1. The bulk of the statistical weight for detecting inflationary B modes is concentrated at two angular scales on the sky (see Fig. 1): firstly, at the reionization bump at multipoles $\ell=2-10$ and secondly at the multipole region from about 20 to 100 (corresponding to angles larger than $\approx 1^{\circ}$ on the sky). Given that most of the signal lies on large angular scales, a full-sky survey with exquisite stability and control of systematic errors of both instrumental and astrophysical origin is required, hence the need to go to space. The $\mathrm{B}$ mode polarization is a clean probe of gravitational waves, since primordial scalar perturbations do not contribute to B-modes, and the effects due to intervening gravitational lenses are calculable and of order $5 \mu K \cdot \operatorname{arcmin}$. This sets the sensitivity target for the mission. In fact, as shown by the blue dotted line in Fig. 2, the lensing contribution to the B-mode is below the primordial B-mode for tensor to scalar perturbation ratios, $r=T / S$, above few $\times 10^{-2}$ at least at multipoles $\ell \lesssim 100$, while, for example, a lensing 


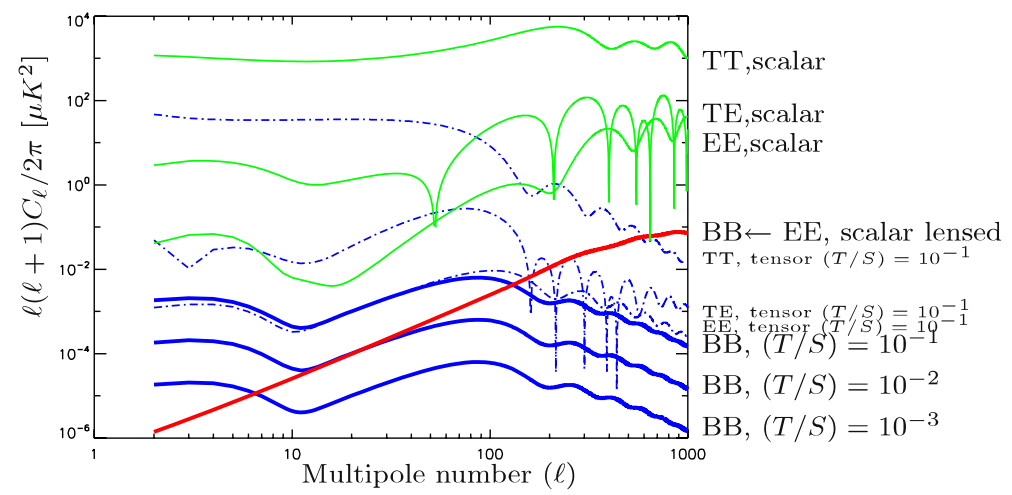

Fig. 1 Inflationary prediction for the CMB temperature and polarization anisotropies, for the scalar and tensor modes. The horizontal axis indicates the multipole number $\ell$ and the vertical axis indicates $\ell(\ell+1) C_{\ell}^{A B} /(2 \pi)$ in units of $(\mu K)^{2}$, which is roughly equivalent to the power spectrum per unit of $\ln \ell$. The green curves indicate the TT, TE, and EE power spectra (from top to bottom) generated by the scalar mode assuming the parameters from the best-fit model from WMAP three-year data. The BB scalar component (indicated by the heavy red curve) results from the gravitational lensing of the EE polarized CMB anisotropy by structures situated mainly around redshift $z \approx 2$. The top four blue curves (from top to bottom on the left) indicate the TT, $\mathrm{TE}, \mathrm{BB}$, and $\mathrm{EE}$ spectra (BB is the heavy solid curve) resulting from the tensor mode, assuming a scale-invariant $\left(n_{T}=0\right)$ primordial spectrum and a tensor-to-scalar ratio $(T / S)$ of 0.1 . This value corresponds roughly to the upper limit established by WMAP. The bottom two blue curves indicate the tensor BB spectrum for $(T / S)$ equal to 0.01 and 0.001 , respectively. For the TE crosscorrelations we have plotted the log of the absolute value

subtraction at $\sim 10 \%$ accuracy level (in terms of angular power spectrum) is adequate to identify the primordial B-mode for $T / S$ above few $\times 10^{-3}$.

A confirmation of inflation and determination of the inflationary potential would have profound implications for fundamental physics by providing new experimental data on the physics near the Planck scale. The constraints established would be indispensable for model building in string and $\mathrm{M}$ theory. The energy scales probed by polarization measurements lie many orders of magnitude beyond any conceivable accelerator experiment. Consequently, the quest for primordial gravitational waves from inflation constitutes a unique window for constraining the new physics near the Planck scale, which will help understand how quantum gravity unifies with the other fundamental interactions.

The implementation of this mission requires significant advances in three main areas: (1) a sensitivity to tensor modes of a factor of about 100 with respect to Planck, (2) control of systematic effects at the level of a few nK, and (3) a precise $(\sim 1 \%)$ knowledge of the galactic foreground polarization. Aspects (1) and (2), related to the B-Pol design, will be extensively discussed in the following sections. Concerning (3), Fig. 2 compares the CMB B-mode (for various $T / S$ values) to the B-mode expected from the most relevant polarized foregrounds and their potential residuals assuming different levels of accuracy 


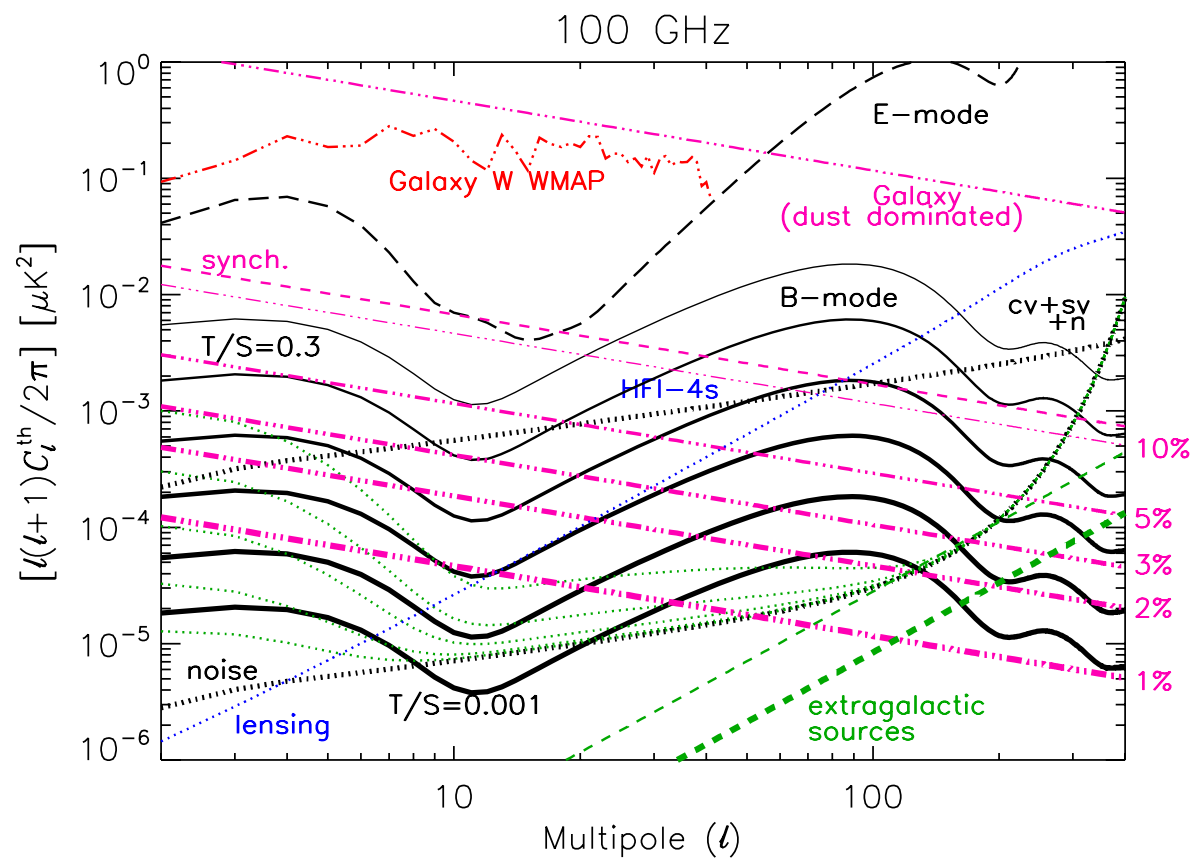

Fig. 2 B mode signal and foregrounds. CMB E and B polarization modes compatible with WMAP 3-yr data are compared to Galactic and extragalactic polarized (B-mode) foregrounds. The anticipated foreground subtraction residuals are compared to the B-mode induced by lensing (blue dots) and to the B-Pol sensitivity. The plots include cosmic and sampling variance plus instrumental noise (green dots labeled with $\mathrm{cv}+\mathrm{sv}+\mathrm{n}$; black thick dots, noise only) assuming a multipole binning of $30 \%$. The B-Pol frequency channel at $100 \mathrm{GHz}$ is considered here. The corresponding Planck-HFI instrumental noise sensitivity is also displayed for comparison (four surveys, upper black thick dots). The $\mathrm{E}$ mode is denoted by the black long dashes. The B mode (black solid lines) is shown for $T / S=0.1,0.03,0.01,0.003,0.001$ from top to bottom, at increasing thickness. Note that the cosmic and sampling ( $74 \%$ sky coverage) variance implies a dependence of the overall sensitivity at low multipoles on $T / S$ (again the green dots refer to $T / S=0.1,0.03,0.01$, $0.003,0.001$ from top to bottom), which is relevant for parameter estimation; instrumental noise only determines B-Pol's capability to detect the B mode. Galactic synchrotron (purple dashes) and dust (purple dot-dashes) polarized emissions produce the overall Galactic foreground (purple three dot-dashes) that is dominated by dust at $100 \mathrm{GHz}$. WMAP 3-yr power-law fits for uncorrelated dust and synchrotron have been used. For comparison, WMAP 3-yr results derived directly from the foreground maps are shown on a suitable multipole range: power-law fits provide (generous) upper limits for the power at low multipoles. Residual contaminations by Galactic foregrounds (purple three dot-dashes) are shown for $10 \%$ to $1 \%$ of the map level, at increasing thickness, as labeled on the right. The residual contribution by unsubtracted extragalactic sources, $C_{\ell}^{\text {resPS, }}$, and the corresponding uncertainty, $\delta C_{\ell}^{\text {resPS }}$, computed assuming a relative uncertainty $\delta \Pi / \Pi=$ $\delta S_{\lim } / S_{\lim }=10 \%$ in the knowledge of their degree of polarization and in the determination of the source detection threshold, are also plotted as green dashes, thin and thick, respectively

for their subtraction assuming B-Pol sensitivities. As is evident, a removal of the foreground, and in particular of the Galactic emission, at the level of about $1 \%$ is necessary to detect the primordial CMB B mode for $T / S \sim 10^{-3}$. Foreground subtraction to this level can already be achieved exploiting already 
(or soon) available all-sky (or large area sky coverage) surveys. Important new data sets will become available in the next years regarding polarized foregrounds in the microwave (e.g., further WMAP surveys, QUAD, BICEPT, EBEX, BOOMERanG, QUIET, C $\ell$ OVER, SPIDER, Planck, etc.), radio (e.g. S-PASS, PGMS, C-BASS, GEM) and far-IR (e.g. PILOT) bands. In parallel, it will be crucial to generalize to polarization the component separation methods successfully applied to temperature data (e.g. Wiener filtering, maximumentropy, Spectral-Matching ICA, CCA, phase methods) as well as to refine the already existing component separation methods for polarized data (e.g. template fitting methods, ICA, FastICA, PolEMICA), and to develop new techniques. Obviously, the broad frequency coverage proposed for B-Pol is crucial to allow the application of these methods at the required level of sensitivity.

Finally we remark that because of its high sensitivity and accuracy in polarization, a mission devoted to B-modes would make substantial contributions in several other areas of astrophysics, such as the physical modeling of Galactic magnetic fields, interstellar dust and gas properties including turbulence effects [16], and of cosmology, such as gravitational lensing of the CMB, cosmological reionization, and magnetic fields in the early universe (see e.g. respectively [17-20], and references therein).

\section{The B-Pol instrument}

B-Pol is a medium class satellite with broad frequency coverage to enable reliable removal of Galactic foreground contamination and an angular resolution good enough to access both multipole windows for detecting primordial gravitational waves from inflation. Accessing the first window requires a full-sky survey, possible only from space. The required sensitivity would nominally require more than 100 years of integration time for the ESA-Planck mission, and moreover much better control of systematics in polarization. To reach the required instrument performances, the detector sensitivity $s_{d e t}$ of $50 \mu K \sqrt{ } s$ can be achieved for an overall instrumental efficiency of 0.5 and a total bolometer NEP (Noise Equivalent Power) of typically $8 \cdot 10^{-18} \mathrm{~W} / \sqrt{ } \mathrm{Hz}$, close to background limited performance. The sensitivity goal in the 6 bands requires a large number of pixels and a long duration mission. Typical values are a total of 2000 detectors for a mission duration of 2 years. The resulting baseline instrumental configuration is summarized in Table 1. For a target sensitivity of $r=10^{-3}$, the r.m.s. signal in primordial B-modes is around $10 \mathrm{nK}$, and rejecting parasitic signals to better than this level imposes very stringent requirements on the polarimeter design and calibration. The strategy for satisfying these requirements is to combine a very stable environment with a carefully designed scan and modulation scheme including redundancies at multiple timescales. In particular, by modulating the polarization signal with a half-wave plate, both $Q$ and $U$ from a given sky pixel can be measured by a single instrument pixel on timescales short compared to the 
Table 1 Main characteristics of the B-Pol instrument

\begin{tabular}{lllllll}
\hline Freq. band $(\mathrm{GHz})$ & 45 & 70 & 100 & 143 & 217 & 353 \\
\hline$\Delta v$ & $30 \%$ & $30 \%$ & $30 \%$ & $30 \%$ & $30 \%$ & $30 \%$ \\
ang. res. & $15 \mathrm{deg}$ & 68 & 47 & 47 & 40 & 59 \\
\# horns & 2 & 7 & 108 & 127 & 398 & 364 \\
det. noise $(\mu K \cdot \sqrt{ } s)$ & 57 & 33 & 53 & 53 & 61 & 119 \\
Q \& U sens. $(\mu K \cdot$ arcmin $)$ & 33 & 23 & 8 & 7 & 5 & 10 \\
Tel. diam. $(\mathrm{mm})$ & 45 & 265 & 265 & 185 & 143 & 60 \\
\hline
\end{tabular}

detector $1 / f_{\text {knee }}$. If we demand that the residual systematics in Stokes maps after correction are less than $10 \%$ of the expected signal from primordial B-modes for $r=10^{-3}$, the uncorrected instrumental polarization (i.e. conversion of total to linearly polarized intensity) due to the polarimeter must be below $10^{-5}$, and the cross-polarization ( $Q$ and $U$ mixing) below $5 \times 10^{-4}$. The latter corresponds to a mis-calibration of polarization angle $<0.03^{\circ}$. Assuming marginalization over absolute calibration errors during parameter fitting, a $5 \%$ uncertainty increases the random error on $r$ by only $10 \%$ (increasing to $60 \%$ if only modes with $\ell>20$ are used). These requirements can be relaxed by additional instrument rotation from a well-chosen scan strategy, as explained below. The experience so far acquired from sub-orbital experiments suggests that the optimal experiment would combine the purity of radiometer frontends and the high sensitivity of bolometers. The B-Pol receivers follow this concept.

A possible implementation of the B-Pol instrument is composed of 8 small telescopes co-aligned with the spacecraft axis. In each telescope's focal plane there is an array of single mode corrugated feed-horns designed to be well matched with the optics and with minimal aberrations. This configuration is sketched in Fig. 3. In Table 2 we report a breakdown of power, volume and mass for the B-Pol instrument.
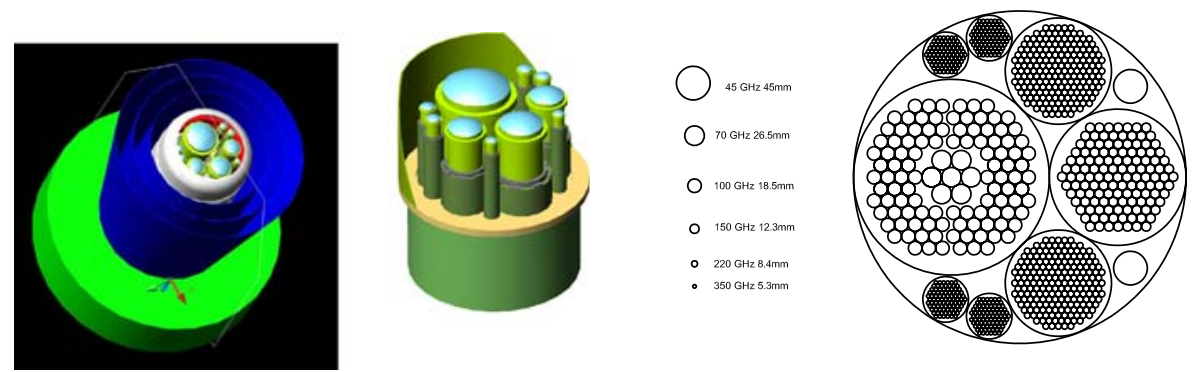

Fig. 3 An artist's conception of the B-Pol satellite (left), of the cryogenic instrument (center), and of the details of the focal plane arrays (right). The cryogenic instrument is enclosed in a $62 \mathrm{~cm}$ diameter, $39 \mathrm{~cm}$ height cylinder, shown here on top of the cylinder enclosing the sub-K refrigerator unit and the cryogenic readout system, which has similar dimensions. The satellite fits in the bay of a Soyuz launcher 
Table 2 Breakdown of B-Pol instrument resources: mass, volume, power

asses and powers include $20 \%$ contingency. The science data produced by the instrument amount to $0.64 \mathrm{Mbit} / \mathrm{s}$, including a mild compression factor

\begin{tabular}{|c|c|c|c|}
\hline Subsystem & Mass (Kg) & Volume (liters) & Power $(\mathrm{W})$ \\
\hline Total sub-K cooler & 20 & 80 & 10 \\
\hline Total focal plane & 74 & 80 & - \\
\hline Horns and focal plane & 29 & & \\
\hline Telescopes & 29 & & \\
\hline Rotation mechanisms & 16 & & \\
\hline Total cryo harness & 5 & 20 & \\
\hline DBU & 13.2 & & \\
\hline BEU & 9.6 & & \\
\hline DHU & 6.6 & & \\
\hline MMU & 9.6 & & \\
\hline PSU & 6.6 & & \\
\hline WPU & 10.1 & & \\
\hline Warm harness & 9.6 & & \\
\hline Total warm electronics & 65 & 40 & 190 \\
\hline Grand-total instrument & 164 & 215 & 200 \\
\hline
\end{tabular}

Polarimetry The incoming polarized radiation is modulated by a single quasioptical Half-Wave Plate that rotates in front of a whole array. Every array pixel consists of a corrugated horn coupled to an Orthomode Transducer (OMT). The OMT cleanly splits the two incoming orthogonal polarizations that are then detected by two TES detectors. OMT and planar phase switches can be fabricated, allowing for intrinsic on-chip polarimetry and therefore eliminating the need for a Half-Wave Plate (HWP) provided that this development is successful during B-Pol phase A. The OMTs are constructed in the waveguide at low frequencies (45 and $70 \mathrm{GHz}$ ) [21] and in a planar microstrip at the higher frequencies $(\geq 100 \mathrm{GHz}) . Q$ and $U$ are extracted by differentiating the detector outputs at different HWP angles. The $45 \& 70 \mathrm{GHz}$ receivers feature a variant of this approach based on a pseudo-correlator scheme, using bolometers as the final detectors.

An achromatic HWP can be made by stacking together several birefringent plates oriented in different directions following the Pancharatnam recipe $[22,23]$. Cross-polarizations of order of $-30 \mathrm{~dB}$ are easily achievable in wide bands using 3 plates. An alternative achromatic HWP uses the same photolithographic techniques adapted for submillimeter mesh filters in order to fabricate artificial birefringent materials.

A special mechanism is necessary to rotate the HWPs, producing modulation of polarization. To achieve a smooth, continuous rotation, each HWP is mounted on a hollow magnetic bearing. These devices have already been used in space (in supporting flywheels for attitude control systems). The rotation is induced by custom made spin-up superconducting motors similar to the type described in [24].

A completely symmetric planar OMT can be built using four probes inside a circular waveguide which are then recombined. Broadband four-probe antennas OMT have been built and tested in the L-band. For a 30\% bandwidth the measured return loss and cross-polarization were, respectively, around $20 \mathrm{~dB}$ and $-40 \mathrm{~dB}[25]$. For the $\mathrm{W}$ and $\mathrm{D}$ bands niobium planar antennas are 
supported by a very thin $\mathrm{SiN}$ layer that is held by a thicker Si substrate etched away to fabricate the waveguide and the probes. The return loss and the cross polarization across the bands are less than $25 \mathrm{~dB}$ and $-50 \mathrm{~dB}$, respectively. Broadband waveguide OMTs are used for the two lower frequencies 45 and $70 \mathrm{GHz}$. Return loss and isolation of order $20 \mathrm{~dB}$ and $50 \mathrm{~dB}$, respectively, have already been achieved at $100 \mathrm{GHz}$ for a $30 \%$ bandwidth [26], and 40 and $70 \mathrm{~dB}$ at $30 \mathrm{GHz}$ [27].

Detectors The baseline requirement is to achieve at all wavelengths highfidelity polarimetry over $30 \%$ bandwidths with NEPs of $8 \cdot 10^{-18} \mathrm{~W} / \sqrt{ } \mathrm{Hz}$ in each polarization, which ensures a CMB photon noise limited sensitivity. Superconducting Transition Edge Sensors (TESs) are ideal for this purpose. TESs have been developed extensively for astronomical observations at millimeter through x-ray wavelengths and have a long history of successful use. Systems using various materials can be combined, and through the proximity effect, bilayers (e.g., $\mathrm{MoCu}, \mathrm{MoAu}$ ) chosen to match the power handling and cooling requirements of the instrument. For B-pol we have selected microstrip-coupled TESs, where a thin-film waveguide probe is used to couple radiation from a horn antenna onto the TES through a superconducting microstrip transmission line terminated with a resistor. With this configuration, corrugated horns can be used to achieve high optical efficiency with low cross polarization, with the significant advantage that it is possible to calculate precisely the optical performance of the whole instrument. Superconducting planar bandpass filters can be lithographed onto the detector chips, and planar OMTs can be fabricated allowing for intrinsic on-chip polarimetry. Microstripcoupled TESs have already been demonstrated in the laboratory by a number of groups, and we have chosen as our baseline a four-probe, membrane-based architecture of the kind being developed for ClOVER [28]. A very recent measurement on a single pixel of C $\ell$ OVER has achieved an optical efficiency higher than $90 \%$.

Any instrument designed for $\mathrm{CMB}$ polarization studies requires a high ( $>80 \%$ ) in-band transmission of the filter stack and simultaneous rejection of optical/NIR power better than $\sim 10^{-12}$. Re-using know-how developed for the Planck HFI makes it possible to develop a filter stack satisfying these stringent requirements.

Optics Specifications directly relevant to the optical design are $\sim 50$ arcmin resolution, $1 \%$ spillover, $\sim 1 \%$ beam ellipticity, and a $30 \mathrm{~dB}$ maximum crosspolarization. While both reflective/mirror and refractive/lens telescopes could be used to achieve the resolution, a lens-based design has been chosen to minimize the required cold volume for the instrument. To overcome the chromaticity problems that lenses and half-wave plates could cause with our wide frequency span and in order to achieve low aberration, we have subdivided the payload into 8 telescope/focal plane systems. Each telescope covers a maximum of 2 frequency bands of $30 \%$ bandwidth each. A 3-lens, F/1.8 
configuration has been demonstrated by optical ray-tracing to achieve the required performance in terms of cross-polarization, beam symmetry, and size of the usable focal plane. In order to reduce the risk of stray light and systematic effects, each focal plane is populated by $\sim 6 \lambda$ corrugated feed horns, their phase center located at the focal surface of the telescope. Corrugated horns are extensively used in CMB experiments due to their low sidelobes and very high polarization purity (see e.g. $[29,30]$ ). The diameter of the feeds is chosen to minimize edge diffraction and spillover (i.e. $-30 \mathrm{~dB}$ of edge taper for F/1 optics). Modules of 7 horns in a single hexagonal module are used to reduce cost and to build the entire focal plane in a simple way. Different manufacturing methods are available: electroforming, direct machining and platelet structure [31,32]. The horns form a curved focal surface to optimize the illumination of the lens system and reduce aberration. Moreover, each optical system is located in a $4 \mathrm{~K}$ black enclosure (eccosorb-like) to make the optical power coming from the $1 \%$ spillover totally un-polarized. This optical concept is valid for all spectral bands but one. Due to the limited size of the payload, we propose to have only 2 channels at $45 \mathrm{GHz}$ with a limited resolution of $15 \mathrm{deg}$ for foreground separation. These two low frequency channels serve as a full-sky calibration reference to merge with smaller but deeper ground-based surveys, which is carried out efficiently below $60 \mathrm{GHz}$. Two stand-alone corrugated conical horns without resort to lenses is optimized to reduce sidelobes and cross-polar components.

Cryogenics The B-Pol cryogenic system uses the heritage from ISO, Planck, and Herschel European space missions, more specifically, the V-groove passive cooling system, lHe cryostat, and sub-Kelvin coolers. This setup should provide a cooling power of $1 \mu \mathrm{W}$ at $100-150 \mathrm{mK}$ for the detectors and a cooling power of $800 \mu \mathrm{W}$ at $2 \mathrm{~K}$ for the cold electronics and the optics as well as active or passive regulation of the various thermal stages so that the induced systematic effects contribute negligibly to the scientific signal error budget. The cryogenic chain must be compliant with pre-launch operations, withstand launch vibrations, and have overall mass/volume/power consumption compatible with launcher and service module.

In addition to an outer screen that provides a first shielding from the Sun, a passive radiator provides a low-cost initial cooling stage for a wet cryostat. For B-Pol, the power must be radiated along the viewing line-ofsight, which covers almost half the celestial sphere within a few days, i.e., an orientation from 90 to 180 degrees away from the Sun. An industrial study was carried out for the Sampan CNES proposal. With forward V-grooves covering an effective area of a few $\mathrm{m}^{2}$, we can achieve temperatures of less than $50 \mathrm{~K}$ at the last internal V-groove. Unlike the mechanical coolers used in Planck, a wet cryostat provides a more compact and lighter solution, and does not produce vibration and magnetically induced parasitic effects on the detectors. The heritage from ISO and Herschel is important in that respect. Either liquid superfluid helium (lHe) or solid hydrogen $(\mathrm{sH})$ can be used. In 
the Sampan case, an unoptimized configuration (compatible with V-grooves at $80 \mathrm{~K}$ ) with a 2.5 year lifetime consists of typically $88 \mathrm{~kg}$ of $1 \mathrm{He}$ for an overall cryostat mass of $352 \mathrm{~kg}$, or $21 \mathrm{~kg}$ of sH for an overall cryostat mass of $109 \mathrm{~kg}$. Both cryostats have similar dimensions. Two possible options for the sub-K cooler are already at a readiness level suitable for this mission. The heritage from Planck HFI $3 \mathrm{He}-4 \mathrm{He}$ open-cycle dilution system is direct. The system has no magnetic influence on the detectors. A powerful passive isolation of the bolometer array plate can be achieved, as in HFI, by using HoY alloys. It can also provide a Joule-Thomson intermediate stage at $1.6 \mathrm{~K}$, which is useful in the case of a sH cryostat. Adiabatic demagnetization of a paramagnetic salt has some possible advantages over the dilution technique, dispensing with the need for liquid confinement and providing a larger cooling power. The main disadvantage is the need of a strong magnetic field obtained with a superconducting magnet, but such a field can be fully contained by means of a superconducting shield. The problems arising for space applications have already been solved. Adiabatic demagnetization refrigerators with an operating temperature below $0.1 \mathrm{~K}$ have been deployed on rockets for X-ray microcalorimetry.

\section{The B-Pol mission}

The rejection of parasitic signals from the side lobes, together with the need of a full sky coverage and the need to avoid Sun, Earth and Moon emission, led to choosing an L2 orbit for Planck. The same considerations apply to B-Pol. A Soyuz launcher that would take off from Kourou offers the most cost effective solution compatible with the instrument requirements. A Lissajous orbit around L2 is preferred to a halo orbit because it is easier to control. It can be phased to avoid eclipses for the entire duration of the mission and can cope with larger payload masses. The mission would last 2 to 4 years depending on the actual cryogenic performance. The transfer from Earth to L2 would last 2 to 4 months. The Calibration and Performance Verification Phase would last 2 months. To achieve optimal sky coverage and redundancy (both in terms of hits per pixel and angular coverage), B-pol uses a complex scan that consists of a precession about the anti-solar axis and a nutation about this precession axis, very much like the WMAP mission. These two motions, with adequately related rotation periods ensure a large sky coverage $(\sim 50 \%)$ over a short timescale $(\sim 2$ days $)$ which together with a shorter $(\sim$ seconds $)$ polarization modulation is optimal for $1 / f$ noise and instrumental drifts rejection. Inflight calibrations are required to correct for gain, polarization angle, differential gain of bolometer pairs, and contamination factors for each receiver ( $T$ into $Q \& U ; Q$ and $U$ mixing). Such calibration is achieved using a set of polarized and unpolarized sky sources observed throughout the entire mission. Onboard artificial sources are also envisaged for more frequent calibration transfer. 


\section{Conclusions}

The search and discovery of primordial B modes provide a unique window for exploring new physics near the Planck scale. There are no competing experimental probes able to access this domain. We have presented an implementation of a CMB polarization mission called B-Pol that fits within the tight requirements for medium-size missions within the ESA Cosmic-Vision 20152025 program. The instrument would survey the sky from the Lagrangian point L2 of the Sun-Earth system for two years, and produce maps of the polarized microwave background anisotropy with sensitivity to $(T / S)$ two orders of magnitude better than Planck. B-Pol would explore the entire parameter space spanned by the "large-field" inflationary models, and consequently is capable to measure the energy scale of inflation. If, as suggested by current CMB and large scale galaxy surveys, the power spectrum of primordial perturbations is not exactly scale invariant, then in a wide class of inflationary models the level of gravitational waves will lie well within the range probed by B-Pol.

\section{References}

1. Starobinsky, A.A.: Relict gravitational radiation spectrum and initial state of the universe. Phys. Lett., B 117, 175 (1982)

2. Starobinsky, A.A.: Relict gravitational radiation spectrum and initial state of the universe. JETP Lett. 30, 684 (1979)

3. Mukhanov, V.F., Chibisov, G.V.: Quantum fluctuations and a nonsingular universe. JETP Lett. 33, 532, 549, astro-ph/0303077 (1981)

4. Guth, A., Pi, S.Y.: Fluctuations in the new inflationary universe. Phys. Rev. Lett. 49, 1110 (1982)

5. Hawking, S.W.: The development of irregularities in a single bubble inflationary universe. Phys. Lett., B 115, 295 (1982)

6. Rubakov, V.A., Sazhin, M.V., Veryaskin, A.V.: Graviton creation in the inflationary universe and the grand unification scale. Phys. Lett., B 115, 189 (1982)

7. Linde, A.: Chaotic inflation. Phys. Lett., B 129, 177 (1983)

8. Bardeen, J.M., Steinhardt, P.J., Turner, M.S.: Spontaneous creation of almost scale-free density perturbations in an inflationary universe. Phys. Rev., D 28, 679 (1983)

9. Abbott, L.F., Wise, M.B.: Constraints on generalized inflationary cosmologies. Nucl. Phys., B 244, 541 (1984)

10. Kolb, E.W., Turner, M.S.: The Early Universe. Addison-Welsey, Redwood City (1990)

11. de Bernardis, P., et al.: A flat universe from high-resolution maps of the cosmic microwave background radiation. Nature 404, 955-959 (2000)

12. Stompor, R., et al.: Cosmological implications of the MAXIMA-1 high-resolution cosmic microwave background anisotropy measurement. Astrophys. J. Lett. 561, L7 (2001)

13. Spergel, D.N., et al.: First-year Wilkinson microwave anisotropy probe (WMAP) observations: determination of cosmological parameters. Astrophys. J. Suppl. 148, 175 (2003)

14. Spergel, D.N., et al.: Three-year Wilkinson microwave anisotropy probe (WMAP) observations: implications for cosmology. Astrophys. J. Suppl. 170, 377, astro-ph/0603449 (2006)

15. The Scientific Programme of Planck: ESA-SCI, vol. 1. astro-ph/0604069 (2005)

16. Cho, J., Lazarian, A.: Angular spectra of polarized Galactic foregrounds. NewAR 47, 1143 (2003)

17. Lewis, A., Challinor, A.: Weak gravitational lensing of the CMB. Phys. Rept. 429, 1 astro$\mathrm{ph} / 0601594$ (2006) 
18. Lesgourgues, J., et al.: Probing neutrino masses with CMB lensing extraction. Phys. Rev., D 73, 045021, astro-ph/0511735 (2006)

19. Burigana, C., et al.: CMB polarization constraints on radiative feedback. MNRAS astro$\mathrm{ph} / 0712.1913$ (2007, in press)

20. Scannapieco, E., Ferreira, P.: Polarization-temperature correlation from a primordial magnetic field. Phys. Rev., D 56, 7493, astro-ph/9707115 (1997)

21. Boifot, A.M.: Classification of ortho-mode transducers. Eur. Trans. Telecommun. Relat. Technol. 2, 503-510 (1991)

22. Pisano, G., et al.: Achromatic half-wave plate for submillimeter instruments in cosmic microwave background astronomy: experimental characterization. Appl. Opt. 45, 6982 (2006)

23. Savini, G., et al.: Achromatic half-wave plate for submillimeter instruments in cosmic microwave background astronomy: modeling and simulation. Appl. Opt. 45, 8907 (2006)

24. Hanany, S., et al.: A cosmic microwave background radiation polarimeter using superconducting bearings. IEEE Trans. Appl. Supercond. 13, 2128 (2003)

25. Engargiola, G., et al.: Tests of a planar L-band orthomode transducer in circular waveguide. Rev. Sci. Instr. 74, 1380 (2003)

26. Pisano, G., et al.: A broadband WR10 turnstile junction orthomode transducer. IEEE-MWCL 17, 286 (2007)

27. Peverini, O.A., et al.: Orthomode transducer for millimeter-wave correlation receivers. IEEEMTT 54, 2042 (2006)

28. Audley, M.D., et al.: Prototype finline-coupled TES bolometers for CLOVER. SPIE 6275E, 69A (2006)

29. Villa, F., et al.: High performance corrugated feed horns for space applications at millimetre wavelengths. Exp. Aston. 14, 1-15 (2002)

30. Maffei, B., et al.: Study of corrugated Winston horns. Proc. SPIE 5498, 812-817 (2004)

31. Kangas, M., et al.: A modular $100-\mathrm{GHz}$ high-gain scalar corrugated nonbonded platelet antenna. Antennas and Wireless Propagation Letters 4, 89-92 (2005)

32. Haas, R.W., et al.: A submillimeter wave platelet horn array: fabrication and performance. In: Proc. 5th Int. Conf. Space and Terahertz Tech., pp. 674-681. MI (1994) 